\title{
XXXVI. Die Gattung Restrepia H. B. U. Kth.
}

(Originalarbeit.)

Von R. Schlechter.

Uls J. Eindley im Jahre 1858 die Gaftung Restrepia für die „Folia Orchidacea" bearbeitete, proteftierte er dagegen, dafiz fihon damals eine $\mathfrak{U}_{n j a h l}$ von $\mathfrak{U}_{\text {rten }}$ in die Gattung eingereiht worden feien, die $10=$ wohl habituell, wie in der Struftur der Blüten ftarł von dem $\widetilde{C}_{\text {Ypus }}$ der Battung, $R$. antennifolia $\mathfrak{h}$. B. u. Kth., abweidhen. $\mathbb{E}_{\mathfrak{r}}$ behielt 9 Zrrten bei der Gattung, von denen aber dte beiden Iekzten, als

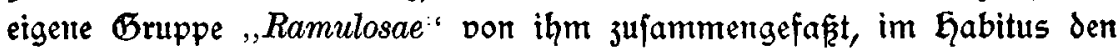
anderen gegenüber recht bedeutend abweidhen. Wie recht viele der damals nod fleinen Batturgen, ijt Restrepia injwijhen ju redht anjehnlidien Dimenfionen angemahjen, denn heute beläuft fíh die Jahl der als Restrepia bejhriebenen $\mathfrak{U}$ rten fhon auf über vierzig.

Zlbgejehen davon, daß̈ von Reidgenbad fil. auch in feinen fpäterent Sebensjahren nod Urten als Reftrepien angefehen wurden, die von Sindley aus der Battung entfernt waren, wie $R$. ujarensis $R$ dqb. $f$. und $R$. ophiocephala $R$ hb. f., fo enthielt dodh die Battung aub fonft noh eine groke Jahl von Cypen, die fhon habitull feineswegs mit dent äbereinftimmen, was der Begründer der Gattung, K. S. Kunth, unter ihr verftanden hatte.

Wir haben uns leider bei den tropifhen Ordideen fehr daran gemöhnt, einen befonders ftarf ausgebildeten habituellen polpmorphismus bei den einzelnen Battungen anzuneḩmen, wie es 3. B. die größzeren amerifanifhen Battungen Pleurothallis, Epidendrum, Maxillaria u. a. beweifen, tatjä丸lid aber wird in vielen fällen der genauere Dergleidh

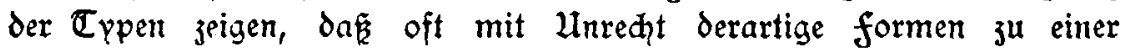
Battung vereinigt worden find. Ja war fo wiederholt bei meinen leķten Urbeiten gejwungen, neue Gattungen ju begründen, die bei genauerer Ultterjudhung der vegetativen und morphologifhen Derhältniffe fhon vor langen Jahren hätten aufgejtellt werden müffen.

Wohl bet Éeiner anderen Pflanzenfamilie find infolge des Jujammen= würfelns heterogener $\mathbb{T}_{y p e n}$ die Gattungsbegriffe fo verworren, wie bei den Ordiideen. Uls Reidenbah fil. peine berühmte und fo äuñerit wertwolle Jufammenftellung für „,Walpers Annales" (vol. VI) anfertigte, war er befonders von dem Gedanfen bejeelt, die Battungen möglidf zufammenjuziehen. Dieles ift von dem nod geblieben, mandies ift wieder getrennt worden. Eeider hat dann Bentham, der' den vegetativen Merfmalen bei den Ordideen merfwürdigerweife fait gar Keine Be*

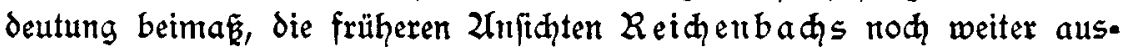


gebaut. Eine Z̈nderung braḑte erft Pfitzer in feiner Bearbeitung der Ordideen in Engler u. Prantls "Ľatürliden Pflanjenfamilien".

In welher $\mathfrak{W}_{e i j e} \mathcal{D}$ fiz̧er die Gattungen weiter getrent hătte, geht jur Genüge aus feinen Bearbeiturgen der beiden Gruppen Diandrae und Coelogyninae in "Pflanzenteiक" hervor, in denen er bei der erften ganj neue und durdaus natürliḑe Begriffe für die Umgrenjung der Battungen fahuf, die fidh jetzt als die einzig riditigen erwiefen haben, während die andere leider nađ feinem Code als fidher niג̧t abgefdiloffene, unfertige $\mathfrak{U}$ rbeit veroffertilidht worden ift.

Es ift felbjtwerftändlid bequemer für den Botanifer groke Gattungen altzunehmell, da er fih darn leidhter und faneller über die allgemeine Derwandt|haft feiner Pflanjen informieren fann, praftifh wird dadurd aber gar nidhts gewonnell, da es dann doh unbedingt nötig ift, grökerere Batturgen weiter in Untergattungen oder Seftionen ju jerlegen. In welher Weife dann dabei gefündigt werden Fann, haben manhe in den lekzten Jahrjebntell eridienenen 2 rbeiten bewiefen, in denen der betreffende Zutor die Urtent nah irgendeinem fünftliđen 2MerPmal zujammenitellt ohne Rüafitht auf ihre natürlidje jülanıntengehörigfeit, und es fo jux ftande bringt, fie derartig bunt durdheinander ju würfeln, dak die $\mathcal{B}_{e s}$ itimmung der $\mathfrak{U}$ rten danad jur Uhmöglidfeit wird.

$\mathfrak{D a}_{\mathfrak{a}}$, wo wir natürliḑe in fĭh gefd̆loffene Battungen befitzen, follelt wir unter allen Umitänden dod ju vernteiden fud,en, durd Ginjufügen heterogener Elemente die Battungsgrenzen übermäp̧ig zu erweitern oder gar 3u veridhleiern, wie es heute leider bei manher grof̈en Ordideengaltung fhon eingetreten ift, in denen fith dann überhaupt nur noh der Spezialift ausfennt, und aud das nur nach überwindung grokerer Shwwierigfeiten. für alle übrigen Bolanifer find diefe Battungen jek̨t fo unüberfithtlid

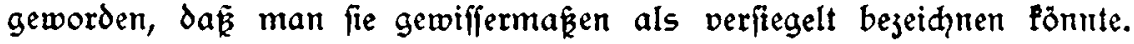

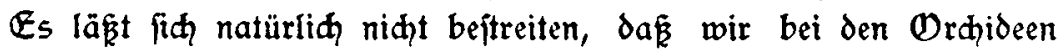
aud einige riefige natürlidie Battungen haben, bei detren über die

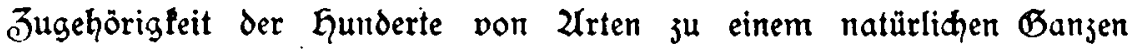

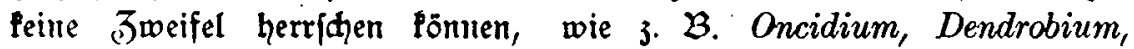
Bulbophyllum u. a., dow aud hier wird die fortfdreitende Erfenntnis der ridhtigen Seftionen und die Zubtrennung befonders ableits fteḩender Eppen noḍ viel jur Erleid̦terung ihrer Einteilung beitragen.

Bei Restrepia liegen die Dinge nun folgendermaken:

Wir haben eine reḑt natürliḑe im Gabitus fowohl wie in der

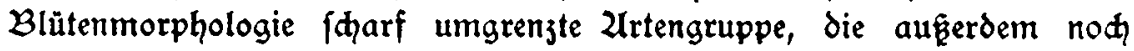
den Dorjug hat, in ihrer Derbreitung auf ein pflanzengeographifh fehr fiharf umgrenjtes Gebiet bef̧ränft ju fein. Daneben finden fid ver. fđiledene Elemente, die frth fowohl habifuell, wie in der Blüte reht gut und leiht unterfheiden laffer, aukerdem aber eine ganj andere $\mathfrak{D e r}$. 
breitung aufweifen. Writ Zusnaḩme von einigen wentigen, nod näher $3 \mathfrak{u}$ unterjudtenden $\mathfrak{A}$ rten bilden dieje abweidhenden $\mathfrak{T}_{p p e n}$ nun eine im Gabitus fowohl wie in den Blütenmerfmalen übereinftimmende Gruppe, die wiedenum für fin betrachtet eine natürlide Gattung darftellt und nah) ihrer Entfernung von Restrepia aud diefer eine fefte Umgrenjung gibt, während beide vereinigt ein unnatürlides Bemijh heterogener Typen darftellen.

Uriprünglidh hatte idh beabfidtigt diefe bei Restrepia herridenden Derhältniffe in einer für fpäter geplanten $\mathfrak{X}_{\mathrm{r} b e i t}$ über die Pleurothallidinase

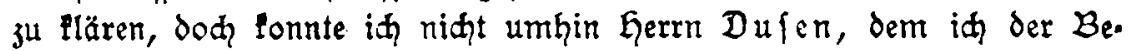
ftimmung feiner von Parana mitgebrachten Ordideent half, darauf auf: merlfam ju mađien. Da er veríhiedene Zurten der braftlianifhen ,, Restrepia ': Gruppe, Jarunter eine $\mathfrak{U}_{n j a h l}$ von Yrovitäten in feiner Sanımlung fant, Iag

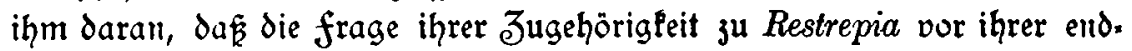
gültigen Bearbeitung entfhieden würde. So habe id, feinem befonderen Wunfthe folgend, fhon jeţt dieje frage zu löjen verfuḑt. Da eine Entficheidung aber erft möglid war nad eirgehender Unterfurhung aller in Betraht fommenden, mir zugänglichen 2 rten, habe ih die ganze Gattung auf die Derwandtfăaft der einzeInen $\mathfrak{Z}_{\text {rten }}$ hin prüfen müfien und gebe als das Refultat hier eine $\mathfrak{U}_{u f j a ̈ h l u n g}$ der bisher befannten Urten.

Da id, nad dem oben Gefagten midh bewogen fühle, Restrepia auf die Urten zu geidränfen, die fih natürlich an Restrepia antennifera h. $\mathfrak{k} . \mathfrak{u}$. Kth., den Typus, aulehnen, fehe id midh aud veranlafit, die abweidhenden Cypen zu einer weiteren Gattung zulammenzufaffen, die, da fie fid in den Blütenmerkmalen und im Gabitus mehr an gewiffe Pleurothallis. Spejies anleḩnt, nad meiner 2 ufjähluug ihren plałz zwijhen Restrepia und Pleurothallis finden foll. Jujammen mit Restrepia untericheidet fie fid von Pleurothallis durd das Dorhandenfein von 4 Pollinien.

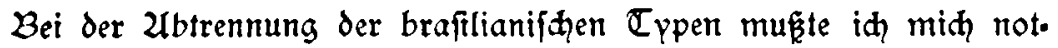
gedrungen aud mit der frage beihäftigen, in welhem Derhältnis Pinelia 3u Restrepia ftehe, denn von Reidhenbad fil. und ihm folgend, von Cogniaux wird fie als zweifelhafte Restrepia.2lrt angejehen. Eindley felbjt fagt bei der Begründung der Gattung, dafi fie im Gabitus an Pleurothallis erinnere und eine Pleurothallidee fein würde, wenn der angefhwollene Stamm nid\}t an gewiffe Epidendra der Aulizeum. Bruppe erinnerte. In felbjt habe auh, midh auf die Zutorität Reidenbahs und Cogniauts verlaffend, eine jweite von Kranjlin ohne befondere $B_{e}$

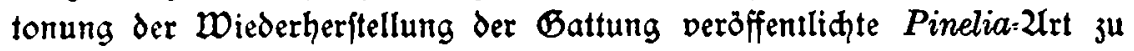
Restrepia übergeführt.

Bei der genaueren Drüfung der Eindleyfhen Befdreibung fiel mir jek̨t auf, daki fie eine ganje Reihe von Charatteren aufweift, die ganj 
entjhịden dafür fprechen, dak̇ hier diefelbe Gattung vorliegt, welthe Rolfe fpäter als Homalopetalum befhrieben hat. Eindle $\gamma$ felbit fagt, $\partial a B_{B}$ er die 2/nthere niht näher unterfuhen Fonnte und deshalb über die pollinien, die nach Pinels Ungaben geldăängt und 4 an der Jahl feien, nähere 2lugaben zu mađhen nidht imftande fei. 2run finden fin befunntlia bei Pinelia vier Pollinien mit Shmänjen, die aber an der Spizze etwas verdiaft find, was wohl von Pinel nidht befonders bemerpt worden ift. $\mathfrak{D a}$ alle übrigen Charaltere von Pinelia vollpommen mit denen von Homalopetalum übereinftimmen, fheint mir die Jdentität der beiden Battungen jum mindejten fehr wahridgeinlid.

Demnad müß̧te Pinelia von den Pleurothallidinae entfernt werden und bei den Epidendrinae, wohin pinel fie fhon verwiefen hatte, ftatt Homalopetalum eingefent werden.

$\mathcal{D a}$ das Eindleyfhe Original jurjeit für mid niḑt jugänglid ift, muk id es anderen ïberlaffen, diefe frage endgültig zu entfheiden. Jedenfalls fheint es mir doh geraten Pinelia junảdfit weiter als eigene Battung ju betrahten.

Bevor id nun dazu übergehe eine $\mathcal{U}$ ufjählung der hier in Betradit fonmenden 2 rten $3 \mathfrak{u}$ geben, will id junadjit auf die Unterfhiede năher eingehen, weldhe mid veranlaffen, von Restrepia die brafilianifhen Zurten und einige meitere auk̇erhalb Brâtliens auftretende abjutrennen. Ih

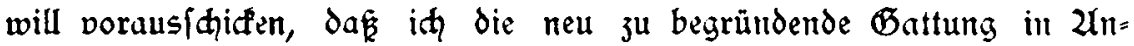
erfennung der Derdienfte des verftorbenen braftlianifinen $\mathfrak{D r d h}_{\text {rddeologen, }}$ 3. Barbofa Rodrigues, deffen Zrbeiten auf dem Gebiete der Ordi $i=$ deologie id oft gellug $z^{u}$ würdigen Gelegenheit hatte, Barbosella be: nenne.

Die edten Restrepia:Urten, ठ. h. diejenigen, welthe fih an $R$. antennifera G. B. u. Kth. anfhliezen, befitzen ftets deutlide, gebüldelte

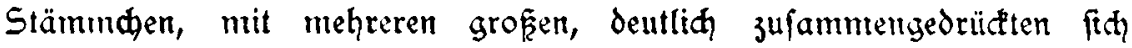
dectenden Sheiden und flahem, lederigifleifhigem, fharfrandigem Blatt, während wir bei Barbosella verlängerte feine Rhizome finden, die itr meḩr oder minder grof̧en ubftänden die auf eincm rudimentären

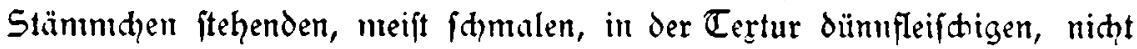
felten ipatelförmigen, meif́ fleinen Blätter tragen. Bei Restrepia zeigen fich die einblütigen Inflorefzenzen gebüldelt, $\partial$. h. die Stämmden bringen aus einer an der Spize ftehenden Sheide mehrere, oft wieder neu erfheinende (iid) alfo nidłt jugleiđ entwidelnde) Blütenftände hervor, während diefe bei Barbosella ftets einzeln und nur einmal entwiafelt werden und nie bie für Restrepia tүpifdęe Sdzeide aufweifen, fondenn nur eine Eleine die Kitofpe urfprünglid fḑüzzende Sdquppe. Die eđ̆ten Restrepia-Zurten haben des weiteren an der deutlih ausgejogenen Spize des mittleren Sepalums und der petalen eine Polbenförmige oder 
antennenartige $\mathcal{U}$ nfhwellung, die fidh bei Barbosella nie findet, infolges deffen ift aubh bei Restrepia das mittlere Sepalum reht verfhieden von den jeitlichen, während es bei Barbosella diejen, abgejehen davon, da范 leţtere aud dort, wie bei Restrepia, verplebt find, red̨t ähnlid ift. Die Sippe ift bei Restrepia durdh den fleifhigen Purzen 2 Tagel feft mit dem Säulenfuke verbunden, daher im lebenden Jujtande niđḥt beweglid, während fie bei Barbosella infolge des dünnen Zagels eine ftarke Bewegliḑleit befitzt, die fith an getrod"́neten Eremplaren nod ftärfer bemertbar maḑt. In iḩrer Gejtalt ijit fie bei Restrepia dadurd charafteriftifh, da die Platte gegen den verdiften Zagel durch eime Querleifte abgejetzt ift, über dem Grunde jwet in eine thah vorn geridhtete pfriemlidge feine Spizze auslaufende Sapper aufweift, während bei Barbosella der über= gairg von der Sippenplatte jum Kagel ein allmählicher ift und die Seitenlappen mur in form dünner Seiften, die nadh der $\mathfrak{E a b e l l u m m i t t e}$ 3u hinablaufen, auftreten. Die Säule ijt bet Restrepia fhlanf mit fapuzen. förmigem Klinandrium und verdiflem, furzen, jehr dharafteriftifhem jederjeits mit einem groken ftumpfer $\mathfrak{G}$ öfer verfeherrem fun

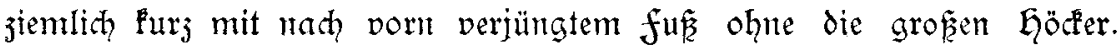

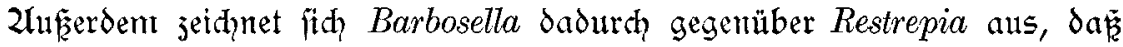
das Rojtellum jungenförmig verlängert und fḑief nad unten vorgeftręt ift, während wir bei Restrepia ein Purzes, fdhief aufreḑtes Roftellum futben. Das Stigma ift bei Restrepia meijt fidmal und ziemlid lang nad unten ausgezogen. Wir habelt alfo jwei Gattungen vor uns, die durdhaus als gut getrennte anjulehen find.

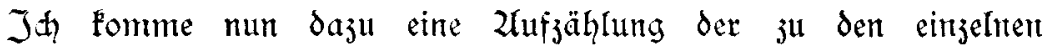
Gattungen gehörenden 2 rten zu geben. Da Barbosella als Gattung hịer neu begründet ijt, jhiffe id) eine Diagnofe der Battung vorauf.

\section{Barbosella Schltr. nov. gen.}

Sepala similia, ligulata, vulgo obtusa, lateralia cohaerentia vel plus minusve adnata, Petala quam sepala plus minusve minora et angustiora, dimidio superiore vulgo leviter angustata, margine saepe minute papillosociliolata. Labellum articulatum, ligulatum, obtusum, retusum vel acuminatum, rarius apiculatum, carinis 2 e basi nunc lobiformibus in medium decurrentibus ornatum, sepalis vulgo multo minus. Columna mediocris, semiteres, subrecta, pede brevi, antice angustato, rostello decurvulo liguiformi, bene longo. Anthera cucullata obtusa. Pollinia 4, oblique clavata vel pyriformia, viscidio parvulo affixa. Ovarium sessile, cylindraceum glabrum.

Plantae parvulae, epiphyticae; rhizomate longe repente, gracili vel gracillimo, radicante; radicibus filiformibus, flexuosis, glabris; caulibus secundariis (i. e. pseudobulbis si licet appellare) abbreviatis, vulgo subinconspicuis, unifoliatis; foliis linearibus vel spathulatis, rarius obovatis 
vel ellipticis, glabris, exsiccatione tenuibus; inflorescentiis gracillime pedunculatis, unifioris, semper singulis, vulgo folia superantibus; floribus minutis vel parvis, rarius submediocribus, illos Pleurothallidis in mentem revocantibus, albidis vel flavidis, vulgo diaphanis.

Species 14 adhuc notae, silvium Brasiliae, Guianae, Ecuadoris, Columbiae nec non Costa Ricae indigenae.

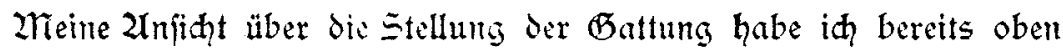
befanntgegebert. Sie mub ohne ङweifel ihren Plat jwifdien Pleurothallis und Restrepia erhalten.

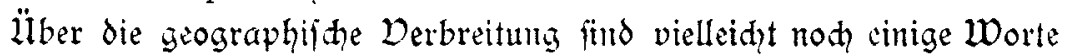
angebrad.t. Uls das Eentrum der Derbreitung ift nad den bisher

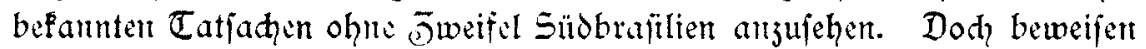
Typen wie B. varicosa (ㅇl.) St)ltr., B. rhynchantha (Rdb. f. u. Warfi.) Saltt., B. cucullata (EOl.) Stltr. mo B. prorepens (Rdb. f.) Sdiltr., da fie aud bis in das anditte Sübamterifa, ja bis Jentralamerifa vor=

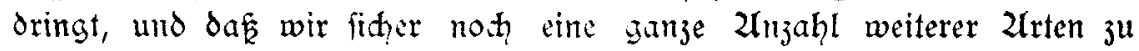
arwarten baben.

Zidįt ausgefdlofien wribcint es mir aud, dafif fid unter den Pleurothallis: $\mathcal{A}$ rten der fogenamiten Prorepentes: Gruppen nod einige Gierber ju redinende 2roten finden. Jedenfalls habe ing die übar= jeugung, dak bic Gattung an 2lrtenjahl balo Restrepia überflüzelt haben wirs.

In dem folgenden wards ith cine 2lufzählung der 2roten mit Furjer Zefpredung ihrer hauptiätidititen 2rarpmale gebent.

1. B. australis (Cogn.) Schltr., n. comb.

Restrepia australis Cogn., in Fl. Bras. III. VI. (1905) p. 564 t. 98 f. 3. Brasilien: Rio Grande do Sul; Sito Paolo.

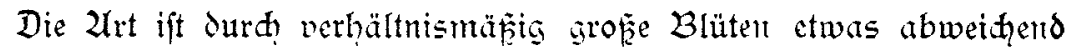
und bildet julfammen mit B. Löfgrenii (Sognt.) Sdiltr. ant beftent cinte cigene Gruppe, bie ourd das vorn ausgejduttene sabellum und die gropen Blüten mit imen nah der Spitse ju papillös behaarten Sepalen

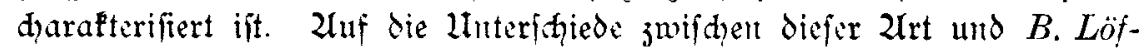

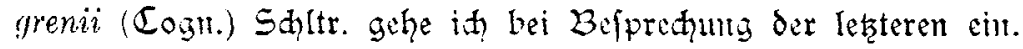

2. B. Cogniauxiana (Speg. u. Kränzl.) Schltr, n. comb.

Restrepia Cogniauxiana Speg. u. Kränzl., in Orchis II. (1908) p: 127 f. 26.

Argentina: Missiones.

Eine jiemlia gedrungene $\mathfrak{U r t}_{\mathrm{rt}}$ der Gauptgruppe, die ith als EuBarbosaea bejeidne, mit furzen elliptifd)=fpatelförnigen Blättern und jiemlid) grozent Blüten auf verhältnismäßzig furzen, die Blätter aller= dings überragenden Săäten. Die Lippe ift als länglid, und ftumpf dargeftellt. 
3. B. crassifolia (Edwall) Schltr., n. comb.

Restrepia crassifolia Edwall, in Rev. Centr. Sci. Comp. no. 4 (1903) p. 193 t. 3.

\section{Rrasilien: Sao Paolo.}

Im Gabitus ähnelt die 21rt am meiften der B. microphylla (Kodr.) Shltr. ift aber leidit von ihr ju unterjheiden durd das dreilappige (?) vorn fehr ftumpfe Sabellum und vollfommen fable Sepalen und Petalen. Die form des Sabellums ijt eine fo abweithende von der der übrigen

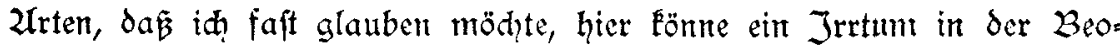
bahtung vorliegen.

4. B. cucullata (Ldl.) Schltr., n. comb.

Restrepia cucullata Ldl,, in Ann. et Mag. Nat. Hist. XV. (1845) p. 108. Columbia: Cauca.

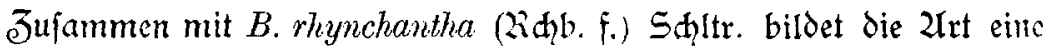
fleine eigene Gruppe, welde ourh den fräftigen $\mathfrak{W}_{\text {uhs, lange falmale }}$ Blätter und anffallend lange Blütenfhäfte harafterifiert ift. Entgegen der Unfiht $\mathfrak{L i n d l e y s}$ glaube id dic beiden beffer als getrente Urtent anfehen zu müffen, auf deren Unterf̧iede iđ unten näher jurüt. fommen werde.

\section{B. Dusenii (Sampaio) Schltr., n. comb.}

Restrepia Dusenii Sampaio, in Arch. Mus. Nac. Rio Jan. XV. (1909) p. 5 c. tab.

Brasilien: Parana.

Ja verdanfe der Güte des Gerrn Dufen Material diefer Seltenheit. Im Gabitus ift fie mit B. Gardneri (Sol.) Shltr. ju vergleidhen, hat aber fürzere, niđht geftielte Blätter, ein länglid)=juntgenförmiges nad der Bafts verfhmälertes Sabellum mit lurjem Spitzhen und jwei furzen

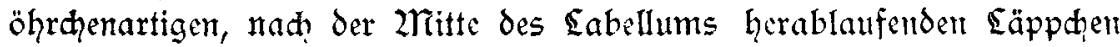

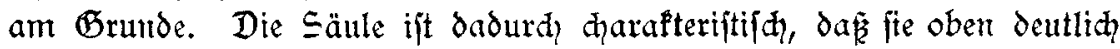
dreilappig ift mit oreizähnigem Mitttellappen (Klinandrium).

6. B. Gardneri (Ldl.) Schltr., n. comb.

Pleurothallis Gardneri Ldl., Bot. Reg. (1842) Misc. p. 83.

Restrepia Gardneri Bth., in Gen. Pl. III. (1884) p. 491.

Humboldtia Gardneri 0. Ktze., Rev. Gen. (1891) p. 668.

Brasilien: Rio de Janeiro; Sao Paolo.

Diefe äußgerft harafteriftifhe Zrt ift durh die form des Sabellums nit den hohen nah der 2ritte herablaufenden Seitenlappen und den ftar jugejpizten 2Mittellappen ju ertemen. Die Blätter find fanmal elliptifh=Lanzettlid, und jemlich Jang und deutlid geftielt. Die Fleinen jarten Blüten ftehen auf haarfeinen, jeḩr langen Sdįften, welḑe die Blätter meift mehr als doppelt ïberragen. 
7. B. Kegelii (Rchb. f.) Schltr., n. comb.

Restrepia Kegelii Rchb. 1., in Linnaea XLI. (1877) p. 133.

Surinam: Mariepaston.

Don diefer 2 rrt habe id lider nur fteriles 2haterial gefehen. Die elliptifacen verfehrteifürmigen, oft faft fpateligen, mit einem fohr furjen

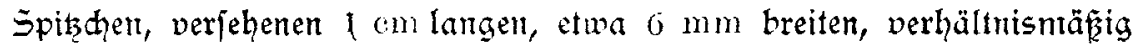
Jiclen Blätter zeidinen fie von den übrigen aus. Die Blütenfhäfte fito uur etwa halb fo lang als die Blätter. Die Make der Blüten hat Zei henbađ leider niht bekannt gegeben. Das Labellum muß ähnlih fonfitruiert fein wie das von B. Gardneri (Sol.) Shltr., foll aber vorn ftumpf fein.

8. B. Löfgrenii (Cogn.) Schltr., n. comb.

Restrepia Löfgrenii Cogn., in Flor. Bras. III. VI. (1905) p. 565.

Brasilien: Sao Paolo.

Wie fhon oben exwähnt ijt diefe 2 rt mit B.australis (Cogn.) Shltr. nahe verwandt. Sie unterf̧eidet fid von ihr ourd lürjere, Wrettere und etwas ditere Blättr, das difere Rhijom, breitere und fürzere Petalen, das brettere Labellum und die fürzere Säule. Jin übrigen ähruelt fith beide 2 rten febr ftart.

9. B. microphylla (Rodr.) Schltr., n. comb.

Res/repia microphylla Rodr., Rev. de Engenh. III. (1881) p. 110.

Brasilien:

In der Struftur der Blüte befingt dic 2lrt eine gewiffe Z̈lhrlidheit mit B. Gardneri (Ed.) Sd)ltr., dod) find die Sepalen und petalen ipitzer und papillös bewimpert und die viel fleineren 3 lätter find męr oval oder längliक und nidyt geftielt. In bell vegetatioen Teilen erimert die Urt an $B$. crassifolia (Eomall) Shitr., die aber Jurd die Eippe fehr gut unteridieden ift.

10. B. Miersii (Ldl.) Schltr,, n. comb.

Pleurothallis Miersii Ldl., Bot. Reg. (1842) Misc. p. 81.

Restrepia Miersii Rchb. f., in Wawra Bot. Erg. Maxim. Bras. (1866) p. 150.

Humbolitia Miersii 0. Ktze, Rev, Gen. (1891) p. 668.

Brasilien: Rio de Janeiro.

Die Pleinfte 2 rt iut ber Battung. Eeider ift das mir ju Behote itehende Material fhon fark verblüht, fo da

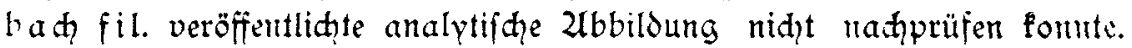
Danad mus fith die 2lrt durh ein auffallend breites $\mathfrak{E}$ abellum aus: jeidhnen. Sie ift aud fonft durd die winjigen Blätter und jiemlia furzen Blütenfääte doaralteriftich. 
11. B. Porschii (Kränzl.) Schltr., n. comb.

Restrepia Porschii Kränzl., in K. Sw. Wet. Akad. Handl. XLVI. no. 10 (1911) p. 51.

Brasilien: Parana.

Die Znt erinnert am meiften an $B$. Gardneri (Edl.) ShItr., ift äußzerlid aber leiđht ju unterjheiden durh die fleineren, fürzeren und verhälmismäf̧ig breiteren Blätter. Die zarten fleinen Blüten ftehen auf Fürzeren Sdïften als bei B. Gardneri (IOI.) Shltr. und die Lippe ift

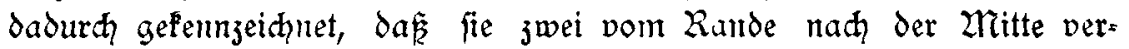
laufende niedrige feine Gautleiften befizt.

12. B. prorepens (Rchb, f.) Schltr., n. comb.

Restrepia prorepens Rchb. f., in Gardn. Chron. (1877) I. p. 810.

Costa Rica:

Dieje äunerit harafteriftifhe Zut dürfte ebenfalls am beften der B. Gardneri ([Dl.) Shltr. Jur Seife geftellt werden, ift aber im ganjen derber mil dideren, fefteren Blütenfhäften und etwas grökeren gelben Blüten, die eiu vorn ftumpfes Sabellum aufweijen.

13. B. rhynchantha (Rchb. f. u. Warsc.) Schltr., n. comb.

Restrepia rhynchantha Rchb. f. u. Warse., in Bonpl. II (1854) p. 114. Peru:

Die 2 rt fheint mir dod von der Folumbianifhen B. cucullata (E১l.) Sdiltr. fpejiffid beffer getrennt zu halten fein. Beide ähreln fid zwar im Gabitus, dod find bei $B$. cucullata (SOl.) Sdltr. die Blüten etwas fleiner, die petalen fhmäler und die rippe jungenförmig, während fie hier unterhalb der Mitte jwei voripringende Winfel zeigt und als fawa oreilappig ju bezeidinen ift.

14. B. varicosa (Ldl.) Schltr., n. comb.

Restrepia varicosa Ldl,, Fol. Orch. Restrep. (1859) p. 3.

Ecuador: Loja.

Diefe 2 rtt ift mir bisher nidit befannt geworden, dod gehört fie nał der vorhandenten Bejhreibung Eindleys wohl ohne Jweifel ju der Gattung. Vielleiḑt gehören fterile Exemplare einter Pleurothallidee hierher, weldie id voll krr. Mille aus Ecuador erhielt. Eindley ver= mutet, dafí die Blütenfärbung orangegelb fei. Die nähere Derwandtfăfit der Pflanze ift jedenfalls nod aufjuflären.

Restrepia H. B. U. Kth.

Nor. Gen. et Spoc. I (1825) p. 367.

Durh die Zusføeioung der oben begründeten Battung Barbasella haben wir in Restrepia ein fehr gut umgrenjtes natürliches Genus vor uns, das in jeiner geographifhen Derbreiturg vollfommen auf das

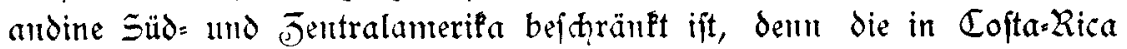


und Guatemala auftretenden Typen fönnen wir ohne Jweifel nod̨ als Zusftrahlungen der andinen flora betradten.

Id) hătte gern, um in der Gattung eimmal eine beffere Überitht

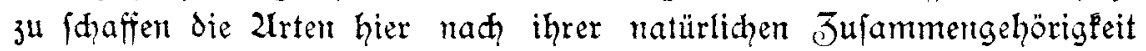
geordnet. Daju wäre aber in einer fo Fritijhenen Gattung wie diefe die erfte Bedingung gewejen, alle Wriginale genau $3 u$ prüfen. Da diejes aber unter den augenblidlidjen Derhältniffen nidht möglith ift, habe id

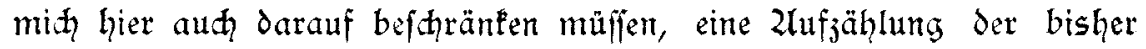
befanntert Zrten ju geben, dod will id fite in Gruppen aufjühlin, wie fie mir natürlidy erfheinen.

Eindey hat in den "Folia Orchidacea" die hier in Betraḑt Fommenden 2lrten in jwet Seftionen geteilt, die "Cirrhosaes und die ,.Ecirrhosae". Diefe beiden Gruppen fämten wohl aud jef̧t nod bejtehen bleiben, dod mülifen wir von den Cirrhosas nod eine fleine Gruppe von Zrten abtremten, die gegenüber allen anderen 2 rten fing durd die

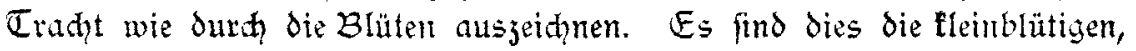

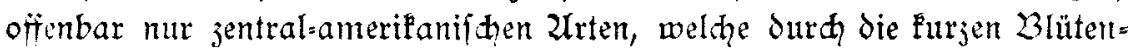
fhaffte, verbältnismäbig Furje Sepalen, fajt ebenjo lange Petalen mit auffallend ftarfer Derdiffung if̨er Spitzen darafterifiert find. Dieje Seftion nemne in, wegen ihrer habituellen z̈hnlid̨leit mit gewiffent Pleurothallis-2Urten, Pleurothallopsis.

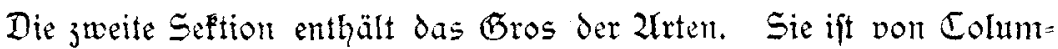
bien und $\mathfrak{W}_{\text {ejt }}$ Denejuela bis nad Ecuador perbreitet. Die $\mathcal{Z}$ rten treten offenbar reht lof́al auf. Charafterifiert ift diefe Seftion durh die lang und ihlank geftielten Blüten uno Jas mit jwei Cirrhen verjehene Eabellum. Jat nenue fie Eurestrepia, da fie den Typus der Gattung einidglię̧冖t.

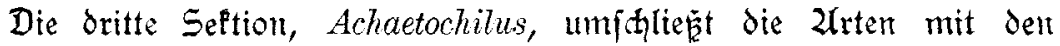
Briten und dem allgemeinen $Z_{11 f b a u}$ der vorigen, aber mit eincr Lippe ohne Cirrher. Das Entuiflunigsjentrum der Gruppe \{heint in den

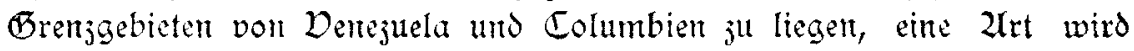
für Eiluador angegeben.

\section{S. Pleurothallopsis.}

1. R. Dayana Rehb. f., in Gardn. Chron. (1875) Il. p. 257.

Costa Rica:

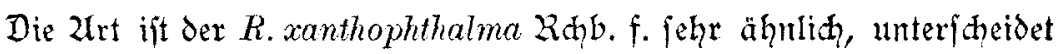
fith aber ourd die fürjeren an Gänge das Blatt faum überragenden Stämmdien mit ftark fḩwarj gefprenfelten Sheiden und das fehr derbe, rundliḑe, am Grunde faft hergförmige Blatt.

2. R. muscifera (Ldl.) Rchb. f., ex Ldl. Fol, Orch. Restrep. (1859) p. 2. Pleurothallis muscifera Ldl., Bot. Reg. (1842) Misc, p. 79.

Guatemala: 
Von den übrigen $\mathfrak{U}$ rten der Seftion unteriheidet fid diefe mir nod

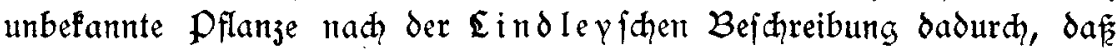
dem Eabellum die bei den anderen zu findenden Cirrhi fehlen.

3. R. Shuttleworthii Rolfe, in Kew. Bull. (1892) p. 133.

Nähere Heimat noch unbekannt.

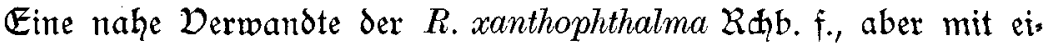
förmigs=länglithen, an der Spitze dreizäḩnigen, $u$ ur etwa $3 \mathrm{~cm}$ langen, etwa $2 \mathrm{~cm}$ breiten Blättern und hellgelben purpurrot gefleften Sepalen und Junfel:purpurn=gefprenfelter $\mathfrak{S i p p e}$.

4. R. xanthophthalma Rchb. f., in Hamb. Gartztg. XX1. (1865) p. 300.

Restrepia Lansbergii Hook., Bot. Mag. (1861) t. 5257 (nec Rehb. f.).

Guatemala:

Eine reizende Fleine 2 rrt mit länglich=elliptichen, am Grunde leil= förmigen, faft fpizen, $4-5 \mathrm{~cm}$ langen, $1,2-2,5 \mathrm{~cm}$ breiten Blättern und hellgelben, auf den Sepalen purpurn=gefledten, auf der Eippe ge, fprenfeltert Baüten.

\section{$\S$ II. Eurestrepia.}

5. R. antennifera H. B. u. Kth., Nov. Gen. u. Spec. I (1825) p. 367 t. 9. Columbien: Nariño.

Die Spejies ift oft mit einer anderen, R. maculata $\mathfrak{E} \delta I_{\text {, }}$ verwed felt worden, die aber im lebenden Jultande durd? ihre gefleffen, nidht ge:

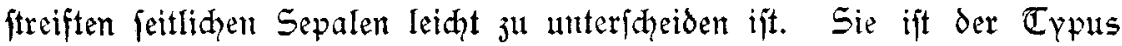

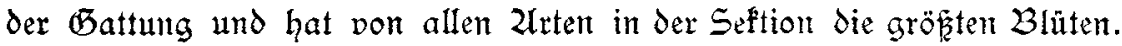
Dieje find bräunlidh=gelb mit purpurn geftreiften Sepalen. Die im ..Botanical Magazin" auf $\mathbb{T}_{a f e l} 7930$ abgebildete Pflartze ift ebenfalls nidht die edte $R$. antennifera $\mathfrak{h}$. B. u. Kth., fondern eine eigente $2 \mathfrak{r t}$, R. Hemsleyana Sdltr., die fidh fiejififh durdiaus von dem Typus unter:

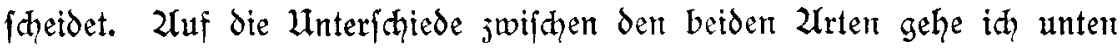
näher ein.

6. R. brachypus Rehb. f., in Flora LXIX. (1886) p. 554.

Colombia:

wie der Spejiesname befagen foll, zeidhnet fid diefe 2 rt durh die Furzen Stämmḑen aus, die deutlich Fürzer find als die feilförmigsläng. lidhen ftumpf:fpiłzlidhen Blätter. Die Sippe weift 5 Sinien von kurjen fpitzliden warjen auf und hat einen aus fd̆mälerem Grunde jungent= förmigen, vorn zweizähnigen Dorderlappen. Reidgenbah fil. gibt leider nidht an, ob die Sepalen geftreift oder geflects find.

7. R. dentata Rolfe, in Kew Bull, (1892) p. 208.

Colombia:

Dieje 2Urt erbielt ith erft vor Furjem als eine Einführung von Colombia. Sie geḩort $\mathfrak{z}$ den 2 rrtent mit geftreiften Blüten und jeidhnet

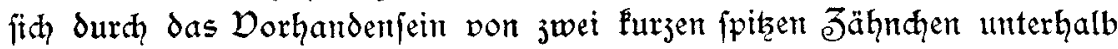


der 2ritte am Rande der petalen aus. Die Eippe ift lältglid, in der 2ritte leiđt eingefdinürt. Die färbung der feitlidien Sepalen ift trüb. gelb mit braumroten Zierven.

8. R. ecuadorensis Rolfe, in Kew Bull. (1892) p. 208.

Ecuador:

Eine der gefledten 2Irtent aus der näheren Derwatdtihaft der $R$. maculata Ed. aber mit fdimäleren am Grunde weniger gerundeten Blättern, fürzeren Blüteniduäften, nid)t geflectem, fondern geftreiftem mittlerem Sepalumt, aber auf gelblith:bräurlidem Grunde, diht braut= gefleften jeitliden Sepalen und ähnlid geflefter, diḅt papillöjer, am Kande wimper:zähniger, in der Mitte leiht veridguälerter Sippe.

9. R. elegans Karst., in Allgem. Gartztg. XV. (1847) p. 202.

Restrepia punctulata Ldl., Fol. Orch. Restrep. (1859) p. 2.

Venezuela: Federal-District, Merida

Unter den Fultiniertell 2 rten ift diefe jeţt entfhieden die häuñgite bei uns. In der Blüte ift fie leiner als die übrigen Zlrten. Das nittlere Sepalum und die petalen find weis, firfhrot geftreift, die feit= lithen Sepalen auf gelbem Grunde diht purpurn gefprenfelt, fpärlid mtit fpitzen Papillen bedectt, in der form länglidh, in der Mitte leidęt ein= geidhürt, an der Spizze furg und ftumpf jweilappig.

10. R. Falkenbergii Rchb. f., in Gardn. Chron. I. (1880) p. 232.

Colombia:

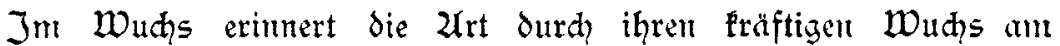
meiften an $R$. antennifera $\mathfrak{G}$. B. $\mathfrak{H}$. Kth., hat aber ungefleatte Sdheiden an denl langen Stämmđhen und mehr keilförmig=länglidhe Blätter. Die Blüten find gejtreift wie bei $R$. antennifera h. B. u. Kith., aber die Eippe ift ftarf geigenfärmig cingejdnürt, mit orei verdiften Kervent, wie fie äbrigens bei verfdiedenen Zluten auftretelt. Die meiften in den botanifonen Girten unter diefem Zamen Pultivierten Eremplare jind, foweit id biss her feftitellen Fonnte, falfh beftimmt.

11. R. Hemsleyana Schltr., spec. nov.

Restrepia antennifera Hemsl., in Bot. Mag. (1903) t. 7930 (nec H. B. u. Kith.).

\section{Colombia:}

Eine der Zrtent mit geiftreiften Blüten. Sie ift als $R$. antennifera im Bot. 2rag. t. 7930 abgebildet, unteridetdet fith aber voll diefer durd) längere Stünmhen, mit fürzeren und daher dihter ftehenden Staciden, etras fleineren didgter geftreiften Blüten und das breitere Eabellum, bei dem die Cirrhi der Eippenbulis mehr genähert find als bei irgendeiner der anderen 2lrten. Die Blätenfärbung ift gelblids mit purpurroten ifimalen Streifen. 
12. R. maculata Ldl., Orch. Lind. (1844) p. 4.

R. antennifera Ldl., Fol, Orch. Restr. (1859) p. 1 (p. pt.).

Venezuela: Merida.

Colombia: Bogota.

Wie id fdion oben ausfüḩrte ift die 2 rnt häıf̆g mit $R$. antennifera h. B. u. Kth. perwedfielt worden, fo j. B. auch in ,Voitch Manual of Orchidaceous Plants". Sie gehört aber $\mathfrak{z a}^{\circ}$ den 2 frten mit gefledten Blüten. Unter diefen jeidhnet fie fỉ aus durh bejonders gropke Blüten. Sie ift auzerdem gut đarafterifiert durd die am Rande fein gejähnelten Sepalen und petalen und die am Rande fein gezähnelte Säule.

13. R. pandurata Rehb. f., in Gardn. Chron. (1888) I. p. 244.

Colombia:

Im habitus befitzt diefe $2 \mathfrak{r t}$ eine gewiffe Z̈hnnlidłeit mit $R$. elegans Karft, dodh find die Blüten größzer und jehr gut gefennzeidnnet durdh das Eabellum. Das mittlere Sepalum und die Petalen find durhylheinend weir mit Firihtoten Streifen, die feitlichen Sepalen auf hellgelbem Grunde diht purpurrot gefleft. Die geigenförmige Lippe ift in eine fajt quadratifhe vorn furj jweilappige Platte bedeutend ftärper verbreitert als bei allen anderen $\mathcal{Z}$ rten. Jhre färbung ift faft goldgelb mit jahlreichen purpurnen Tüpfeln und drei befonders nad vorn erhöhten Zerven.

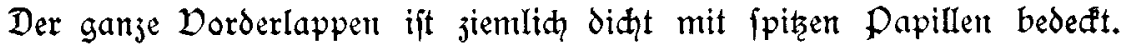

14. R. Schlimii Rehb. f., in Linnaea XLI. (1877) p. 45.

Colombia: Santander.

$\mathcal{R}$ eidenbad fil. vergleidqt die 2 rrt mit $R$. elegans Karft. und $R$. maculata $\mathfrak{E} \delta$. (bei $\mathfrak{i h m ~} R$. antennifera) deshalb fönnen wir wohl att=

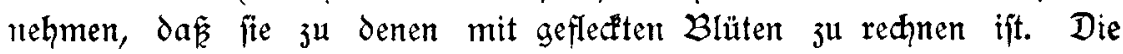
Stämmđinen follen länger fein als die Blätter und mit bandartigz(quer=) gefleçten Sheiden bedeçt fein. Das Labellum wird als feilförmig:länga lidh=zungenförmig, vorn ausgefhnitten beidhrieben und foll nah vorn mit

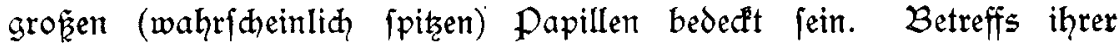
näheren Derwandtfĭaft bedarf die 2lrt jedenfalls nod̆ der 2lufflärung.

15. R. sanguinea Rolfe, in Kew Bull. (1896) p. 44.

Colombia:

Vor allen übrigen 2 lrten ift dieje dadurh ausgezeiḑnet, $\delta a \tilde{k}$ die

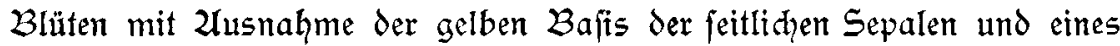
gelben flecfens am Säulengrunde einförmig purpurrot gefärbt find. Die Stämmdhen follen furz fein und ungefleclfe Sheiden befizzen. In den ïbrigen Charafteren wird die mir nodh unbefunnte Spejies als eine Derwandte der $R$. pandurata Rahb. $\mathfrak{f}$. bejeidnnet.

16. R. striata Rolfe, in Gardn. Chron. (1891) I. p. 137.

Colombia: Cauca.

Eine jehr harafteriftifhe $\mathfrak{X}$ rt mit geftreiften Blüten. Sie ift fleiner als die anderen nidit gefledten Spejies und hat bis $4 \mathrm{~cm}$ hohe, von 
ungefleften Şheiden bedefłte Stämmhen mit breit=eiförmigen oder ovalen Blättern. Die Blütenihäfte überragen die Blătter und tragen die für die Seftion mittelgropêt, gelben leud̨tendrot.geftreiften Blüten, deren Fleine Sippe didt rotogefprenfelt ift.

\section{$\$$ III. Achactochitus.}

17. R. aspasicensium Rehb. f., in Bonpl. Ill, (1855) p. 70.

Colombia:

Dor den übrigen Zuten der Seftion ift diefe leinht fenntlidy durch die famalen, lanjettlichen Blätter und die fleinen Blüten, die nach dem

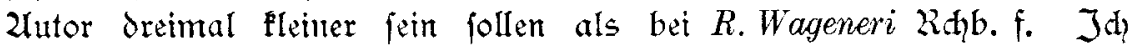
habe die 2 rrt nidht gejehen und bin nidit ganj fidher, ob fie hier ridtig

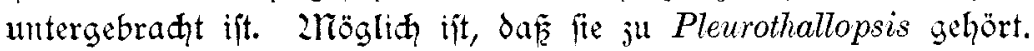

18. R. erythroxantha Rchb. f., in Linnaea XXII. (1849) p. 867.

Venezuela: Merida.

Diefe in der Gruppe reht harafteriftifhe 2 rat zeidhnet fith durdh die fallanfen, das längliḑe Blatt faft doppelt an Gänge überragenden Stanme und auffallend grope feitlihe Sepalem aus. Die fleine Sippe ift auf furz feilförmigem Grunde länglidh:zungenförmig, an der Spitze geftutzt, in den Rändern fein gejähnelt, oberjeits mit Purzelt pitzen $p_{a}=$ pillen bedects. Die petalen und das mittlere Sepalumt find rötlith, die feitlidien Sepalen und das sabellum gelbrot.

19. R. guttulata Ldl., in Hk. Comp. Bot. Mag. II. (1836) p. 357.

Ecuador:

Eine noth aufjuflärende 2urt. Zadh sindley foll fie in allen Teilen der $R$. antennifera h. B. . Kth. (gemeint ift, wenigftens was die fürbung anbetrifft, wohl fid)er $R$. maculata $\mathbb{E} \delta \mathrm{I}$.) gleiḑen, fich aber $\delta \mathfrak{a}=$

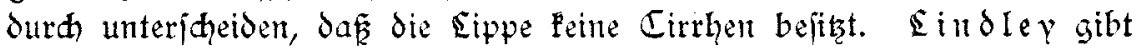

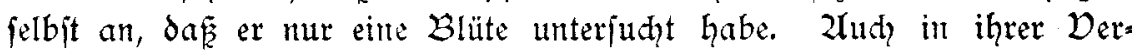
breitung tritt dieje Zurt auperhalb des Gebietes der Sefftion auf, fo da iii vielleiḑt dod nid)t hierher gehört.

20. R. Lansbergii Rehb. f. u. Wagener, in Bonpl. II. (1854) p. 23.

Venezuela: Federal-Distrikt, Merida.

$\mathfrak{Z}_{m}$ beften iff die $\mathfrak{Z}_{\mathfrak{r t}}$ mit $R$. elegans Karft. im habitus $\mathfrak{z}^{\mathfrak{u}}$ ver= gleiđhen, doch ift fie fhlanfer, mit längeren Stämmḑen und fđ̧ntäleren Blättern. Die Blüter find gelbrrot geflecti, mit rot punftierter cippe. Das Sabelium ift undentith oreilappig mit Furjen ftumpfen Seitenlappen und zungenförmigem ftumipfem, oberfeits rauh.papillöfem, von drei ver.

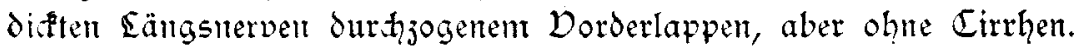

21. R. Wageneri Rchb. f.. in Bonpl. II. (1854) p. 23.

Restrepia Lansbergii Rchb. f. var. Wageneri Ldl., Fol, Orch. Restr. (1859) p. 2.

Venez nela: Merida. 
Der R. Lansbergii Rab̧. f. u. Wagetter fehr ähnlith und etwa gleidh groḱ, aber mit grö̌̈eren Sheiden, breiterem, ant Grunde gerumbetem Blatt und durh die niht gelapple nad Reidgenbah oberfeits Fahle Eippe ausgezeiḑuet.

\section{Noch aufzuklärende Art:}

R. Reichenbachiana Endres, ex Gardn. Chron. (1875) II. p. 356. Costa-Rica:

In fenne diefe Pflanje bisher nur aus Reidhenbahs $\mathfrak{B}_{e}=$ fareibumgen und abbildungen. Danah ift es fehr leitht möglith, fogar

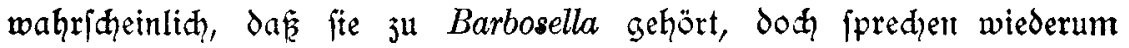
einige 2Merfmale, 3. 23. die Traht, dagegen. Id jiehe deshalb vor die Pflanje noch als zweifelhafte Restrepia:2 $\mathfrak{A}$ t meiterzuführen, bis wir an der hand guten 2liaterials ihre verwandtidhaftlide Stellung gencu fejte legen k̈̈nnen.

Aus der Gattung zu entfernende Arten ${ }^{1}$ ).

1. R. biffora Regel, in Ann. Sci. Nat. ser. 4. VI. (1856) p. $373=$ Plewrothallis hemirhoda Ldl.

2. $R$. (?) hypolepta (Ldl.) Rchb, f., in Walp. Ann. VI. (1861) p. 204 $=$ Pinelia hypolepta Ldl.

3. R. Lehmanniana (Kränzl.) Schltr., in Fedde Rep. III. (1907) p. 277 $=$ Pinelia Lehmanniana Kränzl.

4. R. lonchophylla Rodr., Orch. Nov. I. (1877) p. $35=$ Pleurothallis Warmingii Rchb. f.

5. R. nuda Kl., in Allgem. Gartztg. XX. (1852) p. $275=$ Pleurothallis hemirhoda Ldl.

6. R. ophiocephala Rehb. f., in Bonpl. II. (1854) p. 88 .

Pleurothallis ophiocephala Ldl., Bot. Reg. (1838) Misc. p. 34.

Pleurothallis puberula Kl., in Allgem. Gartztg. XXII. (1854) p. 393.

Pleurothallis stigmatoglosa Rchb. f., ex Ldl, Fol, Orch. Pleur. (1859) p. 68.

Mexiko:

Das mir zurzeit ju Gebote ftehende Material weift leider feine

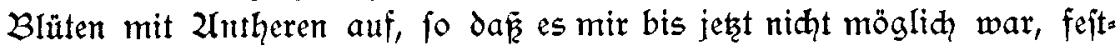
zuftellen, ob die Pflanze zwei oder vier Pollinien befitzt. Sollte fie, wie Reidenbah fil. glaubt brobahtet ju haben, derent vier beftrent, to werden wir wohl faum vermeident fömten, fie als Repräjentanten eirer eigenen Battung anjehen $\mathfrak{z}^{\mathrm{u}}$ müffett.

1) Ich habe hier nur die Spezies angeführt, die nicht unter Barbosella als Synonyme erwähnt sind. 
7. R. parvifolia Ldl,, Orch. Lind. (1844) p. $4=$ Brachionidium parvifolium Ldl.

8. R. pleurothalloides Cogn., in Flor. Bras. III. VI. (1905) p. 566 t. 118 f. 2.

Brasilien: Parana, Sao Paolo.

Ja habe Gelegenhzit gehabt, die Pflunje näher ju unterphden und

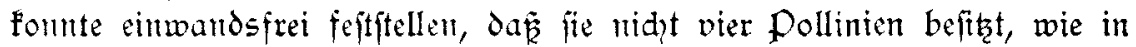
der ..Flora Brasiliensis" altgegeben und abgebildet wird, fondern mur jwei. Sie ift danad, wie aus dem ganjen Gabitus mit der traubigen Infloreszenj ju erwarten war, eine typifdic Pleurothallis=2lrt, die in die Derwandfidiaft von P. bicolor Cogn. ju verwetien ift. $D_{a}$ die Pflanje mumebr einen reuen Zanten erbaltelt mufite, ift fie Pleurothallis Edwallii Dujen u. Shltr. ju nemner.

9. R. tentaculata Poepp. u. Endl., Nov. Gen. u. Spec. I. (1836) p. 34 t. $\mathbf{3} 9=$ Plewrothallis tentaculata Ldl.

$10 R$. ujarensis Rehb. f., in Bonpl. IIl. (1855) p. 225.

Pleurothallis ujarensis Ldl, Fol. Orch. Pleur. (1859) p. 19.

Humboldtia ujarensis 0. Ktze., Rev. Gen. (1891) p. 666.

Costa Rica:

Zlle Blüten des mir vorliegenden von $\mathfrak{X}$. Toubuj aus Coita Rica itammenden :liaterials ind ganj offenbar fleiftogam. Bet allen fehlt das Roftellum, to daj bei Fintreten der Zrarbenfertilität die Pollen= fđlälude fofort auswahjen und die Blüten ftets befruhtet werden. Im übrigen fonte ih die $R e i d$ enbahithen Beobachtungen nachprüfen und fand, datie femc Duritellutig der Pflanje und ibrev 2lnalyfe, $a b=$ gefehen von geriugen Zlbweihungen, durdqaus jutreffent ift. In ben Zntheren fand id vier deutlid anszebildete Pollinien, die paarweife gegeneinander gepreft liegen. Don Pleurothallis muß die Pflanje danach ebenfalls entirnt werden. Ja) lehe aber jetzt noh davon ab, hier eine befondere Gattung anfultellen, da it warten möhte bis Material mit normalen Blüten vorliegt.

11. R. vittata Ldl., in Journ. Hort. Soc. III. (1818) p. $314=$ Pleurothallis hemirhoda Ldl.

\section{Vermischte neue Diagnosen.}

Chaetospermum (Roemer) Swingle in Journ. Wash. Acad, Sci., III (1913), p. 101. - Chaetospermum Roemer, M. J., 1846, Synop. Monogr., I: 39. as subgenus of Limonia. - Type species Limonia glutinosa Blanco, M., 1837, Flora de Filipinas, p. 358. - A genus related to Belou, from which it differs in having persistent leaves with small rounded sessile lateral leaflets, fewer stamens (twice as many as the petals), fewer ovarial cells ( 8 to 10 ), an oblong ribbed fruit with a thick leathery rind and cells lined with a spongy tissue containing many large cavities 